\title{
DGK $_{\zeta}$ Plays Crucial Roles in the Proliferation and Tumorigenicity of Human glioblastoma
}

\author{
Xuefeng Gu ${ }^{1,2,6, \dagger}$, Guoqing Wan ${ }^{1,6, \dagger}$, Nianhong Chen ${ }^{1,6, \dagger}$, Jinhong $\mathrm{Li}^{3}$, Bing Chen ${ }^{4}$, Yeling Tang ${ }^{1}$, Wangxian \\ $\mathrm{Gu}^{1}$, Cuiting Jin ${ }^{1}$, Jihong Meng1, Peng Zhang ${ }^{5}$, Li Liu ${ }^{2}$, Zhifang Yang ${ }^{\circledR}$, Changlian Lu ${ }^{1,6}$ \\ 1. Collaborative Research Center, Shanghai University of Medicine \& Health Sciences, Shanghai, PR China \\ 2. College of Fundamental Medicine, Shanghai University of Medicine \& Health Sciences, Shanghai, PR China \\ 3. Department of Neurosurgery, Jiangmen Central Hospital, Jiangmen, Guangdong, PR China \\ 4. Department of Neurosurgery, Affiliated Hospital of Guangdong Medical University, Zhanjiang, Guangdong, PR China \\ 5. College of Clinical Medicine, Shanghai University of Medicine \& Health Sciences, Shanghai, PR China \\ 6. Shanghai Key Laboratory of Molecular Imaging, Shanghai University of Medicine \& Health Sciences, Shanghai, PR China \\ † contributed equally to this work \\ $\square$ Corresponding authors: 1. Changlian Lu, Collaborative Research Center, Shanghai University of Medicine \& Health Sciences, Shanghai 201318, PR China. \\ Tel.: +86-21-65883218; E-mail: lvcl@sumhs.edu.cn. 2. Zhifang Yang, College of Fundamental Medicine, Shanghai University of Medicine \& Health Sciences, \\ Shanghai 201318, PR China. Tel.: +86-21-65882635; E-mail: yangzf@sumhs.edu.cn \\ (c) The author(s). This is an open access article distributed under the terms of the Creative Commons Attribution License (https://creativecommons.org/licenses/by/4.0/). \\ See http://ivyspring.com/terms for full terms and conditions.
}

Received: 2019.03.25; Accepted: 2019.06.23; Published: 2019.07.20

\begin{abstract}
Glioblastoma is one of the most malignant brain cancers in adults, and it is a fatal disease because of its untimely pathogenetic location detection, infiltrative growth, and unfavorable prognosis. Unfortunately, multimodal treatment with maximal safe resection, chemotherapy and radiation has not increased the survival rate of patients with glioblastoma. Gene- and molecular-targeted therapy is considered to be a promising anticancer strategy for glioblastoma. The identification of novel potential targets in glioblastoma is of high importance. In this study, we found that both the mRNA and protein levels of diacylglycerol kinase $\zeta$ (DGKל) were significantly higher in glioblastoma tissues than in precancerous lesions. The silencing of DGK $\zeta$ by lentivirus-delivered shRNA reduced

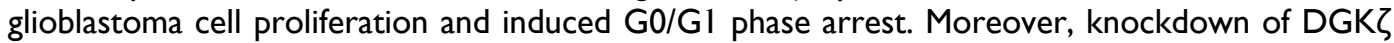
expression in U251 cells markedly reduced in vitro colony formation and in vivo tumorigenic capability. Further study showed that DGK $\zeta$ inhibition resulted in decreases in cyclin DI, p-AKT and $\mathrm{p}$-mTOR. Moreover, the rescue or overexpression of DGK $\zeta$ in glioblastoma cells demonstrated the oncogenic function of DGK . In conclusion, these studies suggest that the suppression of DGK $\zeta$ may

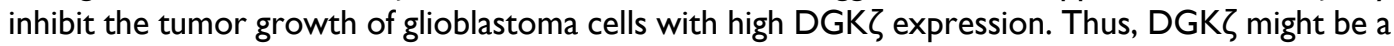
potential therapeutic target in malignant glioblastoma.
\end{abstract}

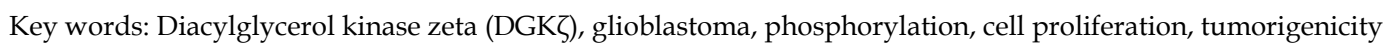

\section{Introduction}

Glioblastoma multiforme (GBM) is reported to be the most malignant brain tumor. It represents approximately $70 \%$ of glial tumors in the central nervous system of humans. Generally, the peak incidence of GBM occurs around 30-40 years of age [1]. GBM is the second greatest cause of mortality in patients younger than 34 years of age. GBM is deadly due to its untimely pathogenetic location detection, infiltrative growth and high recurrence rate. Despite advanced improvements in surgery skill, chemotherapeutic drugs, and stereotaxic radiotherapy, the median overall survival time varies between 12 and 15 months [2]. Therefore, it is very important to find new therapeutic methods to suppress GBM growth and progression.

The functional study of genes or proteins that influence the occurrence and development of GBM will help us understand the molecular mechanisms of the pathogenesis of this fatal disease and identify effective therapeutic methods. Lipids are a component 
of the cell membrane and play an essential role in many cellular events; lipid levels are regulated by lipid-metabolizing enzymes, such as phospholipases and lipid kinases [3]. Diacylglycerol kinase (DGK) is a lipid kinase that catalyzes the phosphorylation of diacylglycerol (DAG) to produce phosphatidic acid (PA), which uses ATP as a phosphate donor [4-6]. Both DAG and PA serve as second messengers in cell signaling. DAG regulates a variety of important signal transducers, including protein kinase $\mathrm{C}$ (PKC) [7] and RAS guanyl releasing protein (RasGRP) [8, 9], while PA plays a key role in regulating signaling proteins such as aPKC, c-Raf, chimaerin [10-12], mTOR [13, 14] and PIP5K [15]. Thus, DGK acts as a molecular hub for many signaling pathways through regulating intracellular DAG and PA levels.

To date, ten DGK isozymes have been reported in mammals, including $\alpha, \beta, \gamma, \delta, \eta, \kappa, \varepsilon, \zeta, \imath$ and $\theta$. These isozymes are divided into five groups (I, II, III, IV and VI) according to their structural characteristics [16]. They exhibit obviously different tissue distributions, expression patterns and biological functions. There is growing evidence that DGKs play an important role in human malignant tumors. For example, several studies showed aberrant expression of DGKa in hepatocellular carcinoma (HCC), mammary carcinoma, melanoma and GBM [17-19], and it is thought to serve as a potential target for drug design [19]. The important crystal structure and properties of $\mathrm{Ca}^{2+}$-bound DGKa-EF were reported by Takahashi [3]. Moreover, increased expression of DGKn was found in malignant lung carcinoma with EGFR or KRAS mutations, and silencing DGKn suppressed tumor cell growth [20]. DGKY plays a tumor suppressor role in HCC and colon cancer by inhibiting cell proliferation and cell migration [21, 22]. It should be noted that diacylglycerol kinases $\zeta$

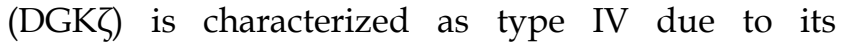
myristoylated alanine-rich C-kinase substrate (MARCKS), ankyrin, and PDZ binding domains [23, 24]. Similar to other DGKs, DGKろ is also reported to be abnormally expressed in human colorectal cancer cells, and it is indispensable for the proliferation of cancer cells. In addition, DGK $\zeta$ knockout attenuated the invasiveness of MDA-MB-231 mammary carcinoma and PC-3 prostate tumor cells [25]. Interestingly, Topham et al. [7] found that the MARCKS domain of DGK $\zeta$ could regulate the nuclear

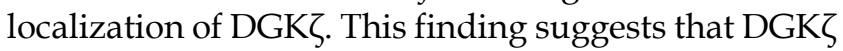
might play different roles in different cellular events. Torres-Ayuso $\mathrm{P}$ et al. [13] reported that DGKל regulates $\mathrm{mTORC1}$ and lipometabolism in human colorectal tumor cells through SREBP-1. Moreover,

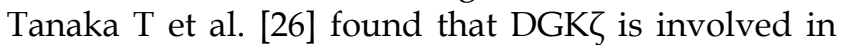
regulating the crosstalk between NF-kB and p53, two major signaling pathways in cell survival and death. Collectively, these findings suggest that regulation of DGK $\zeta$ expression might be an effective way to inhibit the proliferation of metastatic tumor cells, including glioblastoma cells. It will be very important to explore this possibility as there are no studies of DGK了 expression patterns and potential roles in patients with glioblastoma.

In this work, we have presented evidence that DGK $\zeta$ mRNA and protein levels were significantly higher in tumor tissues from glioblastoma patients than in precancerous lesions. Silencing DGK $\zeta$ by lentivirus-delivered shRNA reduced glioblastoma cell proliferation and induced G0/G1 phase arrest. The rescue and overexpression of $\mathrm{DGK} \zeta$ in glioblastoma cells demonstrated the function of DGK $\zeta$ as an oncogene. Moreover, knockdown of DGK $\zeta$ expression in U251 cells markedly reduced colony formation, endothelial cell tube formation in vitro and tumorigenic capability in vivo. Furthermore, western blots showed that the possible downstream molecules of DGK $\zeta$ were phosphorylated mTOR and AKT. In conclusion, these studies suggest that the suppression of DGK $\zeta$ may inhibit tumor development in

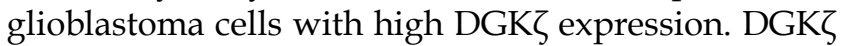
appears to be a potential therapeutic target in malignant glioblastoma.

\section{Materials \& Methods}

\section{Tissue Samples}

Human glioblastoma tissues and corresponding para-carcinoma tissues were obtained from patients who were diagnosed at the Department of Neurosurgery, Affiliated Hospital of Guangdong Medical University (Zhanjiang, China), from 2014 to 2016. The pathological-grade tumors were confirmed according to the WHO 2000 criteria. Normal brain specimens were from victims of automobile accidents without tumors. All patients enrolled in this work gave written informed consent. The protocol was approved by the Ethics Committee of Guangdong Medical University. The clinical samples were washed with normal saline and then immediately frozen in liquid nitrogen for RNA extraction or preserved in $10 \%$ formalin for immunohistochemical staining.

\section{Immunohistochemistry}

Immunohistochemical staining for DGK $\zeta$ was performed on clinical specimens as previously described [27]. The slices were stained with a rabbit polyclonal antibody against human DGK $\zeta$ purchased from LSbio (Seattle WA 98121, USA). Immunoperoxidase staining was performed using an ABC Peroxidase Standard Staining Kit (Pierce, Rockford, IL, USA) according to the manufacturer's 
instructions.

\section{Cell Culture}

U87 MG, HS683, SHG-44, U373, LN229, and U251 cells were obtained from the American Type Culture Collection (ATCC, Manassas, VA, USA). H4 and HEB cells were purchased from Bena Culture Collection (Kunshan, China). According to the supplier's recommendations, all cells were cultured in Dulbecco's modified Eagle's medium (DMEM, Gibco BRL, MD, USA) supplemented with 10\% FBS, 100 $\mu \mathrm{g} / \mathrm{ml}$ streptomycin, and $100 \mathrm{U} / \mathrm{ml}$ penicillin at $37^{\circ} \mathrm{C}$ in a humidified incubator containing $5 \% \mathrm{CO}_{2}$.

\section{DGKろ silencing and overexpression}

We created lentiviral constructs that inducibly express DGK $\zeta$-targeting shRNAs (GeneBank Accession: NM_003646) or control shRNA. The shRNA sequences were as follows: DGK $\zeta$-shRNA1, 5'-TACTCTGAAAGCAAGCAAGAA-3'; DGK shRNA2, 5' - AGTGGTGTGTGATGGAATGGACT-3'; and scrambled, 5'-TTCTCCGAACGTGTCACGT-3'. Lentiviruses were packaged and purified to yield

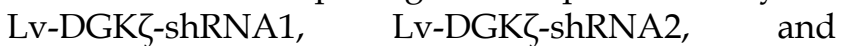
Lv-scr-shRNA, which were used to infect glioblastoma cells. DGK $\zeta$ expression after targeted shRNA lentivirus infection was examined by qRT-PCR or western blot analysis on the fourth day.

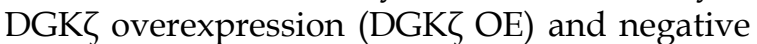
control lentiviral vectors were obtained from GeneChem Co., Ltd. (Shanghai, China). After infecting U251 cells for 4 days, the expression of DGK $\zeta$ was examined by western blot analysis.

\section{RNA extraction and qRT-PCR}

TRIzol reagent (Invitrogen, Karlsruhe, Germany) was used for total RNA isolation. Reverse transcription PCR was performed using a one-step RNA PCR kit (TaKaRa, China). SYBR-Green Master PCR Mix (Applied Biosystems, Life Technologies, USA) was used for qRT-PCR. All qRT-PCR primer pairs were purchased from SABiosciences. Data collection was performed using an ABI PRISM 7500 sequence detection system (Applied Biosystems, Life Technologies, USA). $\beta$-actin was selected as endogenous control to eliminate errors in reverse transcription PCR and to standardize the measurement of RNA expression levels. The relative quantification value for each target gene was compared with the control ( $\beta$-actin), which was expressed as 2-(Ctest-Ccontrol) (Ctest and Ccontrol are the mean cycle threshold $(\mathrm{Ct})$ value differences after normalization to $\beta$-actin). The relative expression levels of the samples were determined by semi-log plot.

\section{Western blot analysis}

Total protein was extracted from glioblastoma cells using RIPA buffer (Thermo Fisher Scientific, Waltham, MA). The protein concentrations were examined using a BCA Protein Quantification Kit (Yeasen, Shanghai, China). Samples containing $30 \mu \mathrm{g}$ protein were subjected to SDS-PAGE and electrophoretically transferred to nitrocellulose membranes (Millipore, Bedford, MA, USA). The membranes were blocked with solution containing $5 \%$ nonfat dry milk and then incubated overnight at $4{ }^{\circ} \mathrm{C}$ with specific primary antibodies, which included DGK了 (LS-C101153), anti-cyclin D1, anti-mTOR, anti-phospho-mTOR (Ser2448), anti-AKT and anti-phospho-AKT(Ser473) (1:1000, CST, Beverly, MA) antibodies, followed by incubation with a secondary antibody (goat anti-rabbit IgG (HRP) from Abcam, ab6721). After the membranes were washed, the blots were developed using an ECL kit (Yeasen, Shanghai, China).

\section{Edu assay}

Infected cells were cultured in 96-well plates and incubated with $50 \mathrm{mmol} / \mathrm{L}$ EdU (RiboBio) for 2 hours. Cells were fixed with $4 \%$ paraformaldehyde for 30 minutes at room temperature. After permeabilization with $0.5 \%$ Triton-X-100, the cells were incubated with fluorescent staining solution for 30 minutes. Then, the DNA in the cells was stained with Hoechst 33342 for 30 minutes. Cells were then observed with a confocal fluorescence microscope (Zeiss, Thornwood, NY).

\section{Flow cytometry analysis of the cell cycle}

U251 cells were cultured in 6-well plates and treated for 48 hours. Subsequently, the transfected cells were collected after washing twice with PBS and then fixed in $70 \%$ ice-cold ethanol at $-20^{\circ} \mathrm{C}$ overnight. The next day, the fixed cells were stained with 50 $\mu \mathrm{g} / \mathrm{mL}$ PI (propidium iodide, ABCONE, Shanghai, China) containing $50 \mu \mathrm{g} / \mathrm{mL}$ RNase A (DNase-free) for 15 minutes at room temperature. Flow cytometric analysis was performed using a flow cytometer (FACSCalibur, Becton Dickinson) and FlowJo software (Tree Star).

\section{Colony formation assay}

For the colony formation assay, U251 and U87 MG cells (500 per well) were seeded in a 6-well plate at $24 \mathrm{~h}$ post-transfection and cultured in medium containing 10\% FBS for 10 days. When the colonies reached more than 50 cells, they were fixed with $10 \%$ formaldehyde for 15 minutes and stained with an $8 \%$ crystal violet solution for 10 minutes; then, the colonies were imaged using a digital camera. Each experiment was repeated three times. 


\section{Tube formation assay}

A 96-well plate was coated with growth factor-reduced Matrigel (BD Biosciences, USA); then, $2 \times 10^{5} \mathrm{HUVEC} / \mathrm{ml}$ in complete medium and $100 \mu \mathrm{l}$ of U251 medium were added to each well. One hundred microliters of $60 \%$ diluted tumor conditioned medium from cells treated with DGK $\zeta$ shRNA1 or shRNA2 was added to each well, and tube formation was monitored with an inverted microscope after 6 hours of incubation. The total tube length in each well was measured and calculated using ImageJ. Experiments were performed in triplicate.

\section{Tumorigenesis assay}

Female nude athymic mice ( 7 weeks old, weighing approximately $18 \mathrm{~g}$ ) were purchased from Shanghai Sippr BK Laboratory Animals Ltd. (Shanghai, China). The mice were maintained in a specific pathogen-free (SPF) animal facility. All animals received humane care according to the National Research Council's guidelines. U251 cells infected with Scr-shRNA or DGKろ-shRNA1 lentivirus were suspended in PBS at a final concentration of $5 \times$ $10^{7}$ cells $/ \mathrm{ml}$. A $100-\mu \mathrm{l}$ aliquot of the cell suspension (approximately $5 \times 10^{6} \mathrm{U} 251$ cells) was injected into the axilla region of nude mice in the treatment and control groups. The mice were sacrificed 28 days post-injection, and tumor growth was examined. The tumor sizes and volumes were determined through external measurements and calculated by the formula: $\mathrm{V}=\left[\mathrm{L} \times \mathrm{W}^{2}\right] \times 0.5(\mathrm{~V}=$ volume, $\mathrm{L}=$ length and $W=$ width). The data were analyzed using Student's t-test, and differences were considered significant at $P$ $<0.05$.

\section{Statistical methods}

GraphPad Prism 6.0 software (GraphPad Software, CA, USA) was used for data analysis. The data are expressed as the mean \pm standard deviation (SD). Student's t-test or one-way analysis of variance (ANOVA) followed by Dunnett's post hoc test was utilized to analyze the differences between the two groups. $P<0.05$ was considered statistically significant.

\section{Results}

\section{DGKろ expression was upregulated in glioblastoma.}

DGK $\zeta$ mRNA expression was first examined in glioma and corresponding para-carcinoma tissues from 44 clinical patients diagnosed with gliomas. The qRT-PCR results showed that DGK $\zeta$ mRNA expression was obviously higher in glioma tissues than in paired normal tissues from most (39 of 44) patients (Fig. 1A and B). Subsequently, immunohistochemical staining was performed to confirm the protein expression of DGK $\zeta$ in the glioma tissues. DGK $\zeta$ levels appeared to be higher in malignant glioma tissues than in precancerous tissues from glioma patients (Fig. 1C and D). However, there was no significant correlation between DGK $\zeta$ and glioma tumor grades because of the limited sample size (Table 1). To further detect the correlation between DGK $\zeta$ expression and glioma, we used eight cell lines, HEB, LN229, HS683, SHG-44, H4, U373, U251 and U87 MG, to examine DGK 3 mRNA expression by qRT-PCR analyses. The qRT-PCR

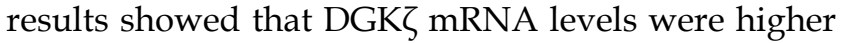
in 4 cell lines than in normal brain tissue samples; U251 and U87 MG cells had the highest expression levels (Fig. 1E). In conclusion, our results suggested that DGK $\zeta$ expression was upregulated in gliomas.

Table 1. Associations between DGK $\zeta$ expression and the clinicopathological characteristics of 44 glioma patients

\begin{tabular}{llllllll}
\multicolumn{2}{l}{$\begin{array}{l}\text { Clinicopathological } \\
\text { characteristics }\end{array}$} & $\mathrm{n}$ & $<0$ & $0-1$ & $1-2$ & $>2$ & $P$ value \\
\hline Age & $\leq 60$ & 36 & 3 & 2 & 16 & 15 & 0.303 \\
& $>60$ & 8 & 2 & 1 & 4 & 1 & \\
Sex & male & 26 & 4 & 2 & 11 & 9 & 0.761 \\
& female & 18 & 1 & 1 & 9 & 7 & \\
\multirow{2}{*}{ Grade } & I-II & 15 & 3 & 2 & 5 & 5 & 0.295 \\
& III-IV & 29 & 2 & 1 & 15 & 11 &
\end{tabular}

$P$ values were detected by the chi-square test.

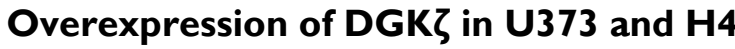 glioma cells enhanced cell proliferation}

Gain- or loss-of-function studies were performed to investigate the potential role of DGK $\zeta$ in glioma cells. First, we overexpressed DGK $\zeta$ in U373 and H4 glioma cells with low endogenous DGK $\zeta$ expression (Fig. 1C, 2A and 2D). The effect of DGK $\zeta$ on glioma cell proliferation was measured using the CCK8 assay. A significant increase in the cell growth curve after transfection with DGK $\zeta$ was found in both U373 and $\mathrm{H} 4$ cells (Fig. 2B and E). This result indicated that cell proliferation was enhanced due to the upregulation of DGKろ. To further confirm this result, we carried out EdU proliferation assays. Consistent with the CCK8 analysis results, both U373 and H4 cells showed higher EdU-positive staining after overexpression of DGK (Fig. 2C and F). These results suggest that DGK plays a role in promoting glioma cell proliferation. 


\section{Knockdown of DGKל in U251 and U87 MG glioma cells inhibited cell proliferation, while rescue of DGK $\zeta$ increased cell proliferation}

Subsequently, high DGK expression in the U87 MG and U251 cell lines was knocked down (Fig. 3A and D). Five days after DGK $\zeta$-shRNA knockdown in the U87 MG and U251 cell lines, CCK8 analysis was performed, and the results showed cell growth inhibition compared with that in Scr-shRNA-infected cells. The viability of DGK $\zeta$-shRNA-infected cells was obviously lower $(P<0.01)$ than that of Scr-shRNA-infected control cells (Fig. 3B and E).

An EdU proliferation assay was employed as an alternative method to measure cell proliferation.
Consistent with the CCK8 analysis results, both U251 and U87 MG cells showed lower EdU-positive staining levels after transfection with DGK $\zeta$ shRNA than after transfection with control shRNA (Fig. 3C and F).

A rescue experiment was used to confirm the

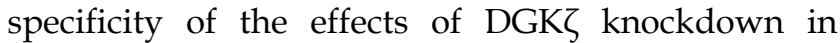
glioma cells. DGK $\zeta$ expression was enhanced after transfecting the DGK $\zeta$ vector in DGKろ-shRNA1-infected U251 cells (Fig. 3G). Furthermore, enhancing DGK expression rescued the decrease in cell viability induced by RNAi-mediated DGK $\zeta$ depletion (Fig. $3 \mathrm{H}$ and I).
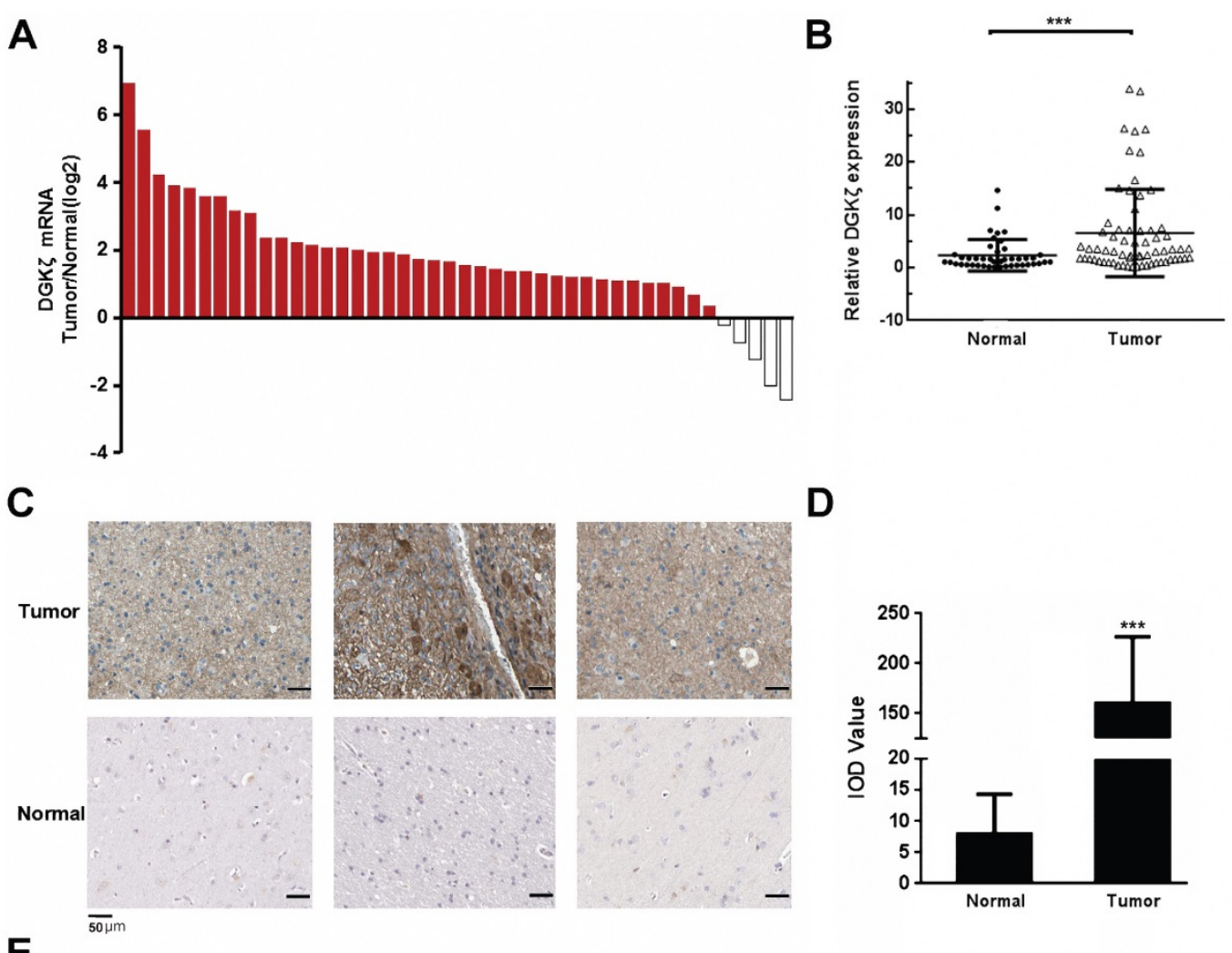

E

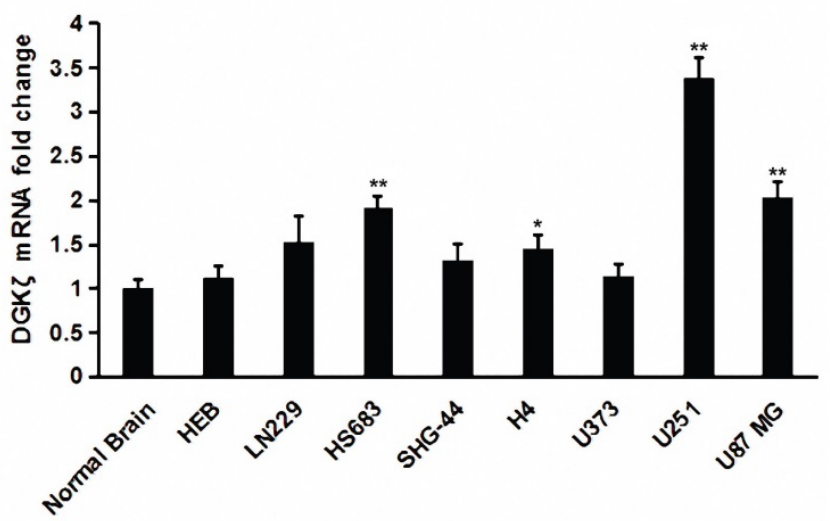

Figure 1. DGK $\zeta$ is highly expressed in glioma cells. (A, B) DGK $\zeta$ mRNA expression was obviously higher in glioma tissues than in paired normal tissues in most patients (39/44). (C, D) Representative immunohistochemical staining for DGKZ expression in human glioma tissues (upper) and precancerous tissues (lower). The average IOD value was obtained by analyzing five fields per slide with Image-Pro Plus software (v. 6.0) and recorded in histograms. (E) DGK $\zeta$ mRNA levels in normal brain tissues and glioma cell lines detected by qRT-PCR analysis. The results represent at least three separate experiments. Error bars: \pm S.D. $* P<0.05, * * P<0.01, * * * P<0.001$. 
A

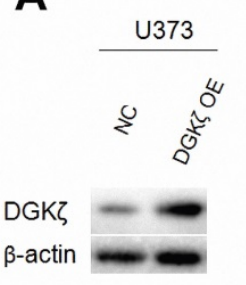

D

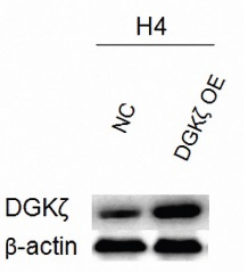

B

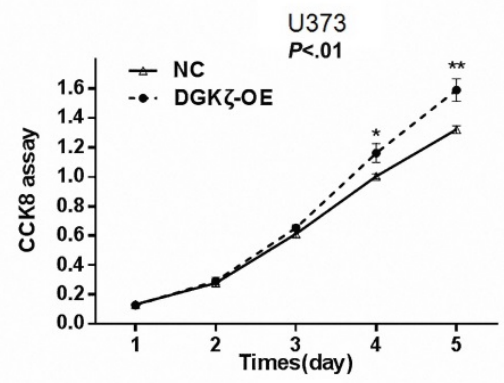

E

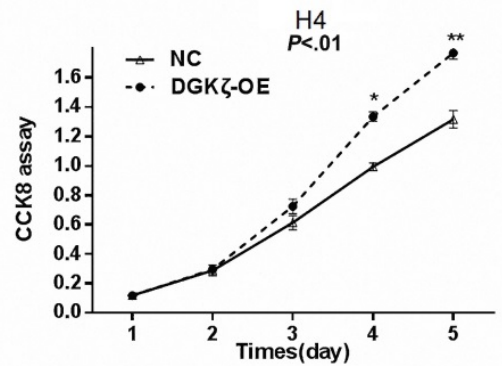

C

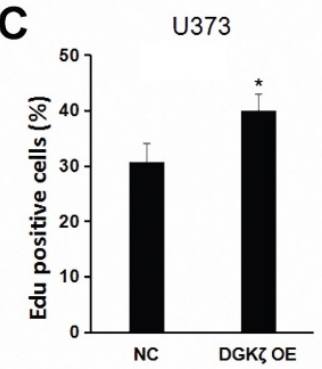

$\mathbf{F}$

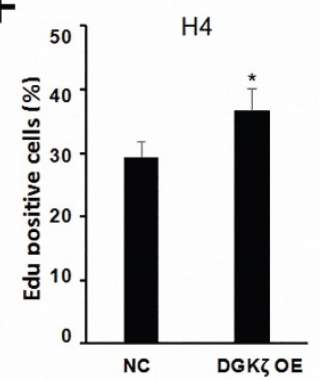

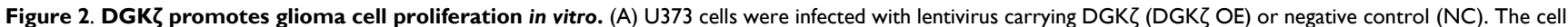

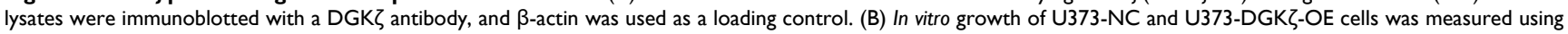

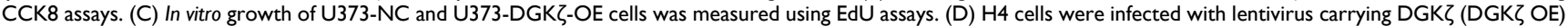
or negative control (NC). The cell lysates were immunoblotted with a DGK $\zeta$ antibody, and actin was used as a loading control. (E) In vitro growth of $\mathrm{H} 4-\mathrm{NC}$ and $\mathrm{H} 4-\mathrm{DGK \zeta}$-OE

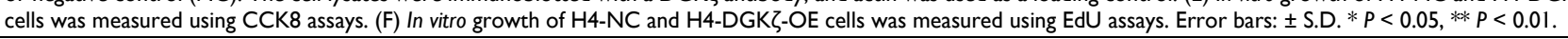

A

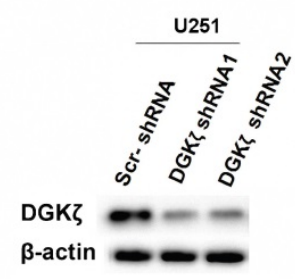

D

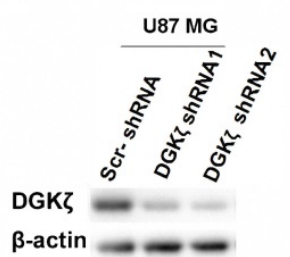

G

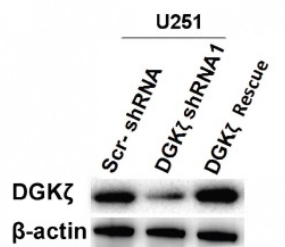

B

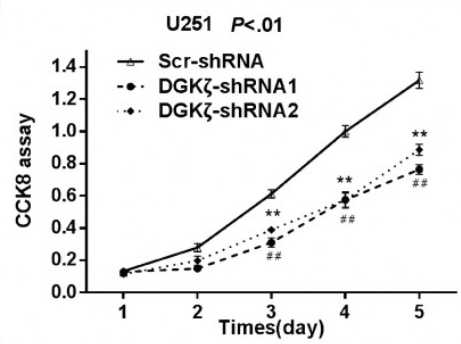

$\mathbf{E}$
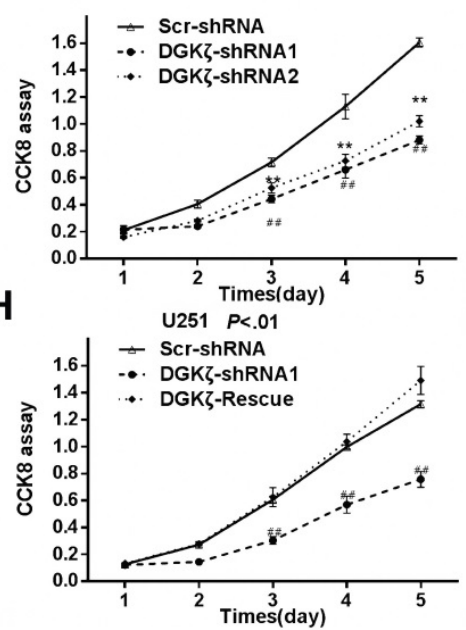

C

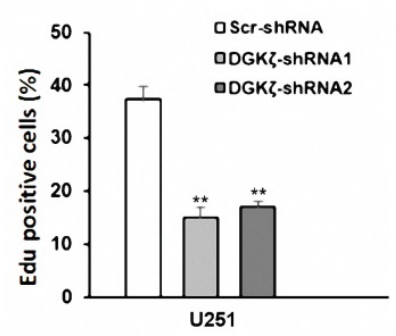

$\mathbf{F}$

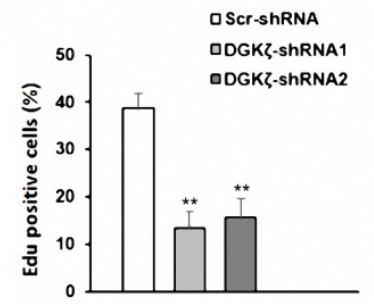

I

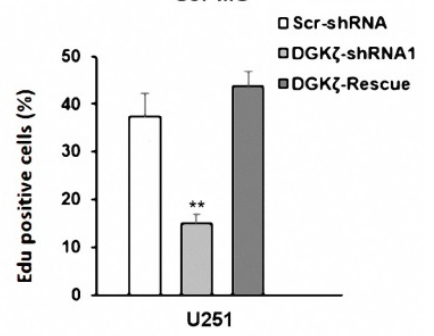

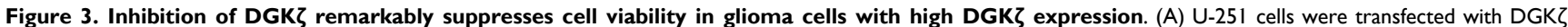

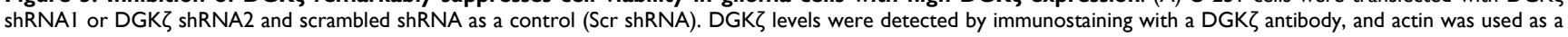
loading control. (B) In vitro growth of U-251/Scr shRNA and U-251/ DGK $\zeta$ shRNA1 or shRNA2 cells was measured using CCK8 assays. (C) In vitro growth of U-251/Scr shRNA

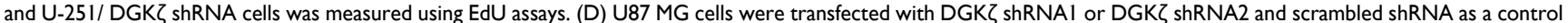

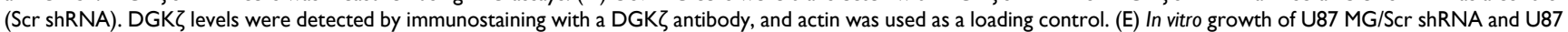
MG/DGK $\zeta$ shRNA1 or shRNA2 cells was measured using CCK8 assays. (F) In vitro growth of U87 MG Scr shRNA and U87 MG/DGK $\zeta$ shRNA cells was measured using EdU assays. (G) DGK

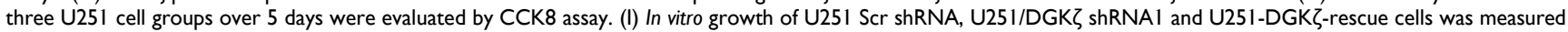
using EdU assays. Error bars: \pm S.D. $* P<0.05$, $* * P<0.01$. 


\section{Inhibition of DGKろ significantly augments the tumor growth of DGKZ-expressing glioma cells}

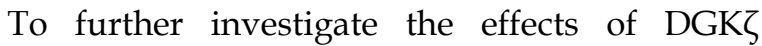
inhibition in glioma cells, colony formation, flow cytometry analysis of the cell cycle, and tube formation assays were performed in vitro. Transfection of U251 and U87 MG cells with DGKל shRNA resulted in a significant decrease in the cell numbers of each colony compared with nonsense shRNA transfection. The number of colonies with more than $50 \%$ cells was also decreased in DGK $\zeta$ shRNA-transfected cells (Fig. 4A and B).

After U251 cell PI staining, flow cytometry

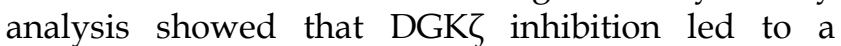
significant increase in G0/G1 phase arrest but a decrease in the number of $S$ phase cells (Fig. 4C and D). These data demonstrated that DGK $\zeta$ inhibition results in U251 cells in the quiescent phase.

Moreover, a tube formation assay revealed that knockdown of DGK $\zeta$ could inhibit the formation of extravascular blood vessels (Fig. 4E and F).

We next examined the in vivo effect of DGK了 using a subcutaneous xenograft nude mouse model. Significant inhibition of tumor growth relative to the control group $(82.8 \% ; P<0.01)$ was recorded in the DGK $\zeta$ knockdown group (Fig. 5). Considering all the experiments together, the most significant result obtained was that the inhibition of DGK $\zeta$ expression suppressed the tumor growth of glioma cells with high DGK $\zeta$ expression.
A

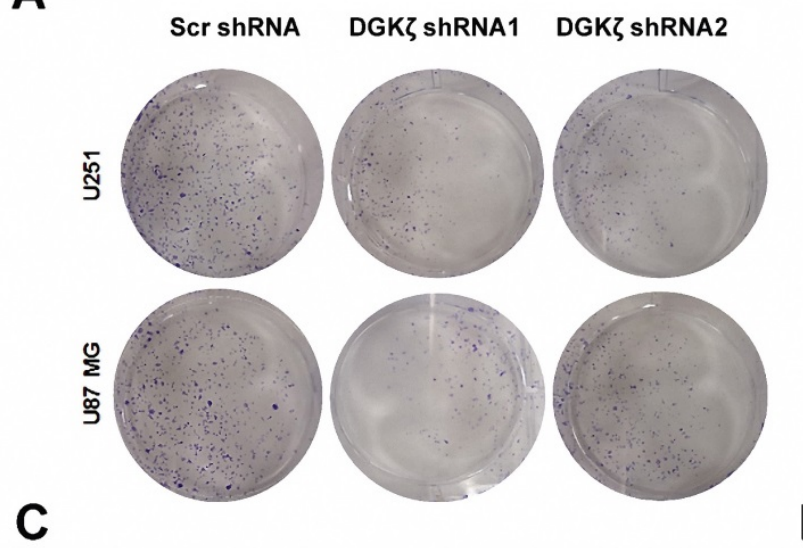

D
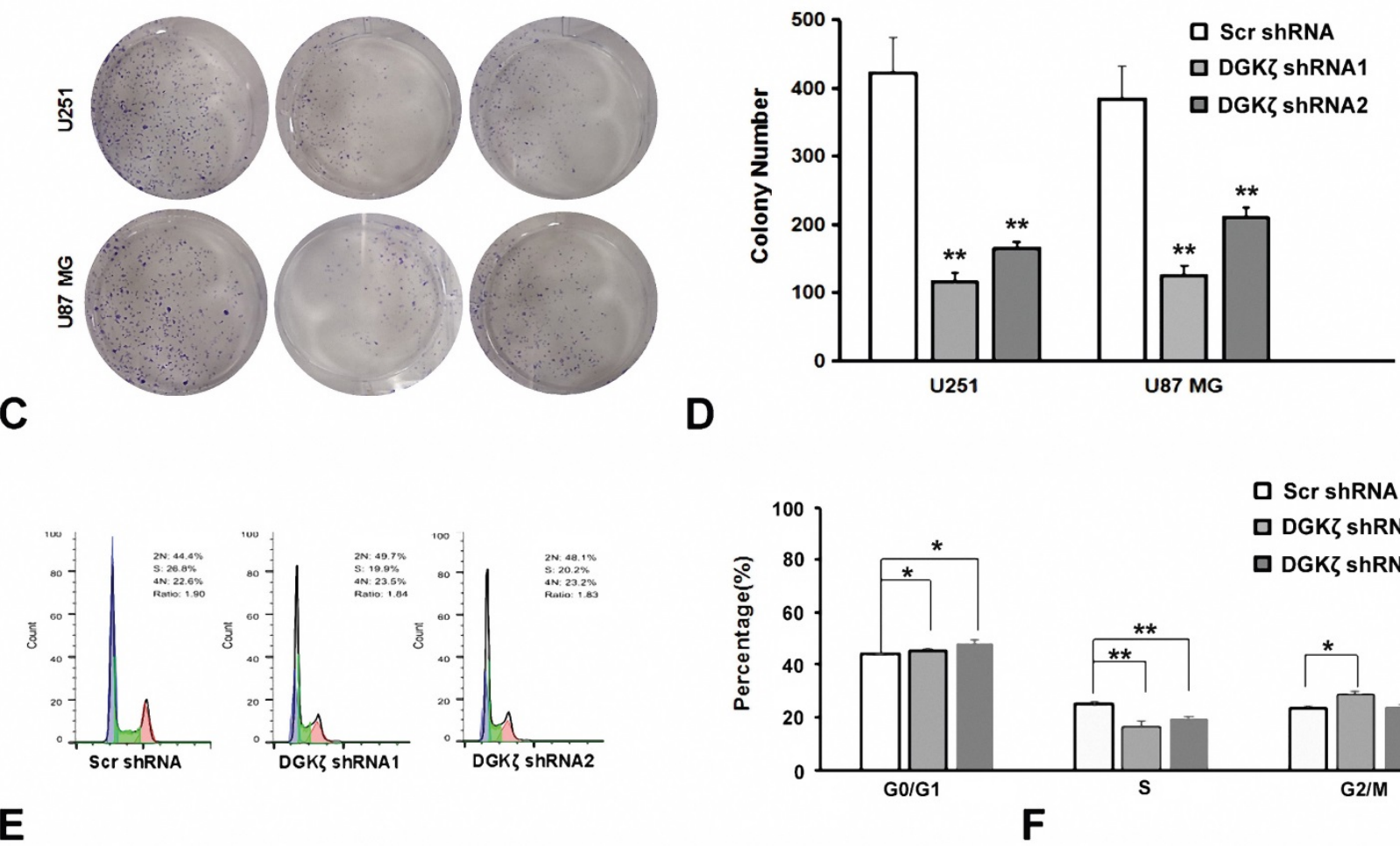

E

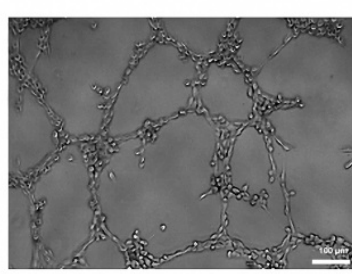

Scr ShRNA

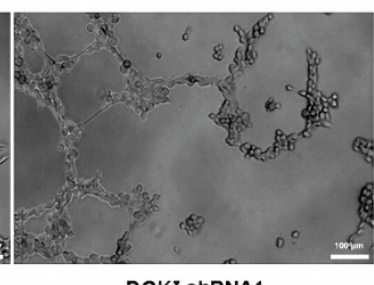

DGKל ShRNA1
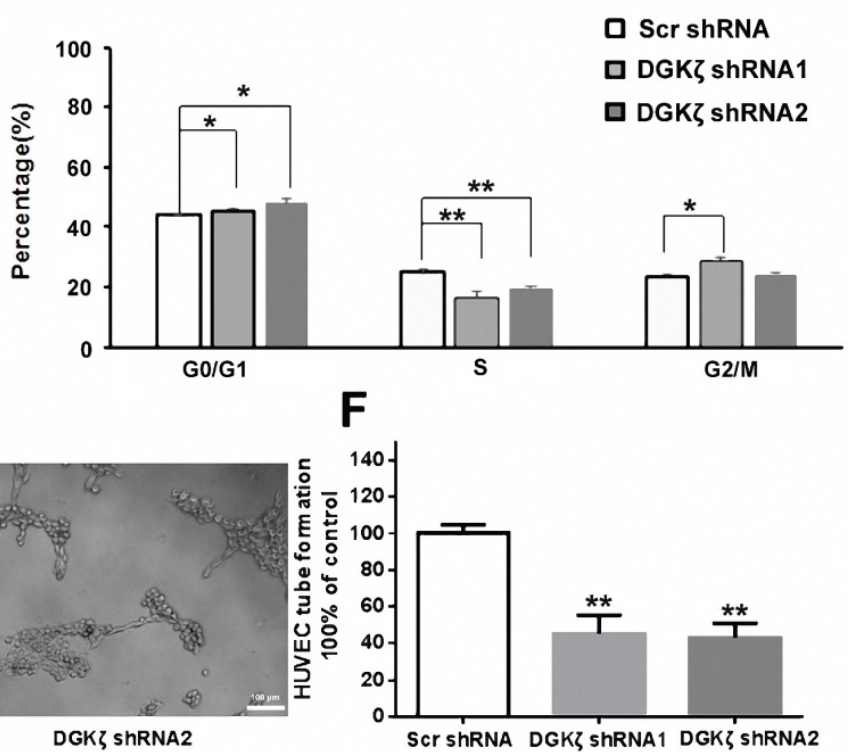

Figure 4. Inhibition of DGKZ prominently suppresses tumor development in glioma cells. (A, B) Colony formation assay of cells from the three groups. Cells were seeded at 500 cells/well and allowed to form colonies for 10 days. The colonies were stained with crystal violet and observed. (C, D) Cell cycle analysis of cells from the three groups as determined by PI staining and FACS analysis (left). The percentages of cells in each phase represent the mean \pm S.D. of three independent experiments (right). $* P<0.05$, $* * P<0.01$. (E, F) Representative images from the HUVEC tube-formation assay. Quantified data correspond to the mean \pm S.D. of three independent experiments (right). $* * P$ $<0.01$. 


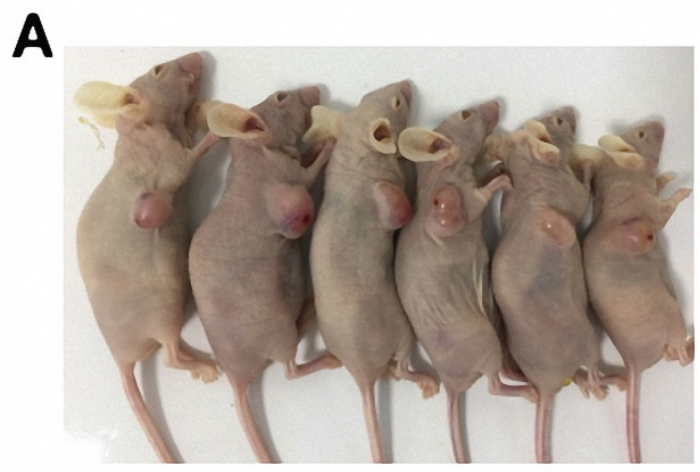

Scr-shRNA

B

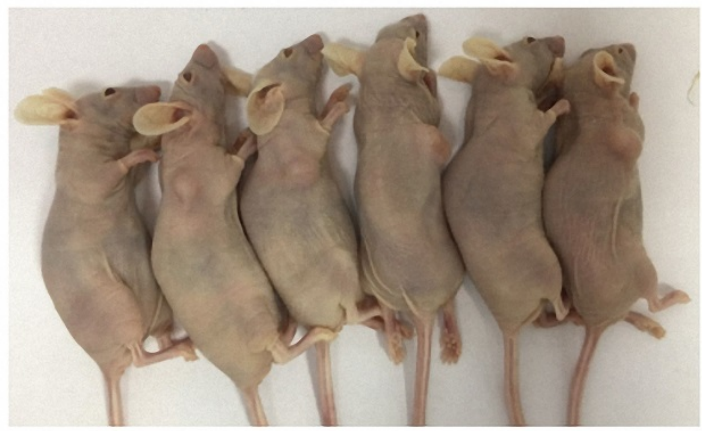

DGKろ-shRNA1

C
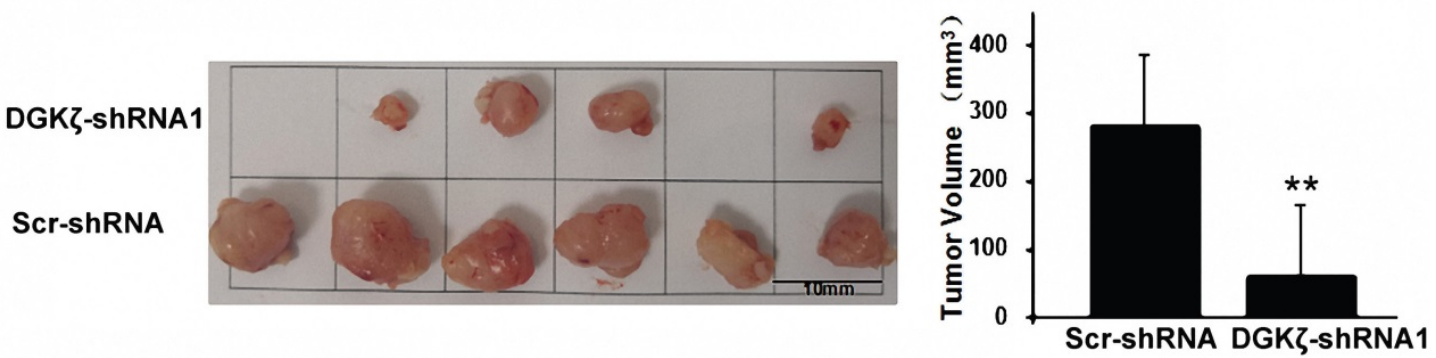

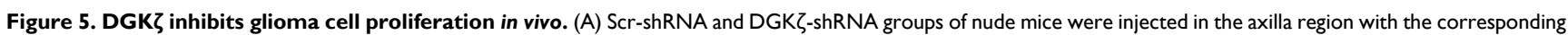
infected $\mathrm{U} 251$ cells $\left(10^{6}\right.$ cells per mouse). After 4 weeks, the mice were sacrificed, and their tumors were examined $(n=6)(B)$. (C) Quantified data correspond to the mean \pm S.D. of two groups $(\mathrm{n}=6)$. $* * P<0.01$.

\section{DGKろ silencing caused the downregulation of cyclin D1, AKT phosphorylation and $m$ TOR phosphorylation}

We next investigated the mechanism by which DGK $\zeta$ inhibition suppressed glioma growth in vitro and in vivo. Because the role of DGK $\zeta$ in carcinogenesis as well as in tumor progression has been poorly documented, only a few studies have reported that DGK $\zeta$ has an anti-apoptotic function that is dependent on the mTOR pathway [28-31]. Moreover, the flow cytometry results showed that U251 glioma cells were arrested at the G0/G1 phase. $\mathrm{PI} 3 \mathrm{~K} / \mathrm{AKT} / \mathrm{mTOR}$ is an important intracellular signal transduction pathway involved in the cell cycle; thus, AKT/mTOR-associated signaling pathways were examined first. Western blot analysis showed that the phosphorylation levels of mTOR and AKT were lower in DGKZ-shRNA-infected cells than in control cells (Fig. 6), while there was no evident change in total $\mathrm{AKT}$ and mTOR, indicating that DGK $\zeta$ silencing inactivated the AKT/mTOR pathway in glioma cells through a phosphorylation/dephosphorylation mechanism. We also found the downregulation of

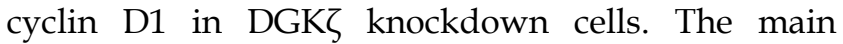
function of cyclin D1 is to promote cell proliferation and G1/S phase transition by binding and activating cyclin-dependent kinase CDK4, phosphorylating cyclin inhibitor protein $(\mathrm{Rb})$ in the G1 phase, dissociating the phosphorylated $\mathrm{Rb}$ protein from its binding E2F transcription factor, and initiating the transcription of genes in the cell cycle. The molecular mechanisms of how these genes work downstream of $D G K \zeta$ require future evaluation.

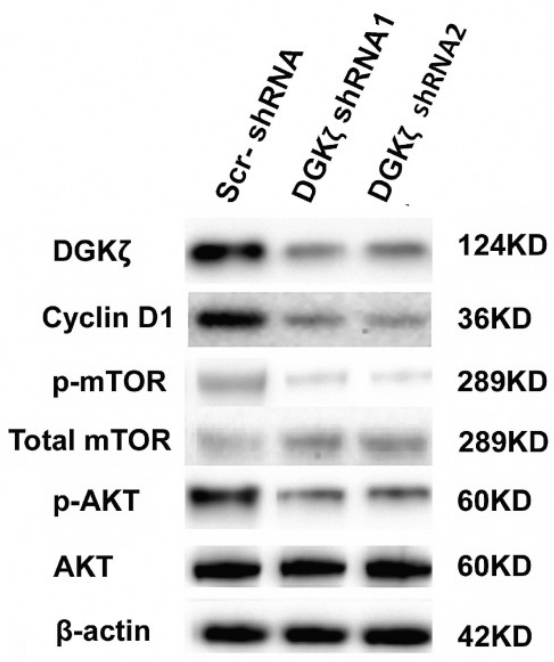

Figure 6. DGKろ silencing decreases cyclin D1, phosphorylated AKT and phosphorylated mTOR levels in U251 cells. U251 cells were transfected with lentiviruses expressing the shRNAs. DGK $\zeta$, cyclin DI, phosphorylated mTOR and phosphorylated AKT protein levels were assayed by western blots. Western blot

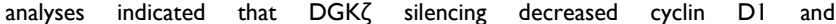
phosphorylated mTOR and phosphorylated AKT protein levels. One representative result is shown. 


\section{Discussion}

Gliomas can occur at any age, regardless of sex and race. Based on their histopathology, gliomas can be subdivided into grades I-IV according to the World Health Organization (WHO) classification for CNS tumors [32]. Grade III and IV gliomas are more aggressive and difficult to cure due to the frequent dysfunction of tumor suppressors and oncogenes. Despite the combination of surgery, radiotherapy, and chemotherapy, the prognosis for patients remains very poor, and less than $5 \%$ of patients with malignant glioma survive 5 years [33]. Presently, the only chemotherapeutic drug is temozolomide; Everolimus is a molecule-targeted drug for subependymal giant cell astrocytoma (SEGA) associated with tuberous sclerosis (TS) that cannot be treated surgically, and Avastin is a monoclonal antibody $(\mathrm{mAb})$ drug that is the only second-line treatment for malignant glioma approved by the FDA [34]. As we better understand the molecular mechanisms underlying glioma development, molecular targeted therapy has been proposed as a promising treatment for glioma. For instance, BRAF [35], EGFR [36], EZH2 [37], MET [38] and mTOR [39] have been reported as potential protein targets for glioma treatment. However, because of the complexity of the occurrence and development of glioma, none of these targets can completely cure glioma; thus, more novel therapeutic targets are urgently needed.

In the present study, we demonstrated that DGK $\zeta$ mRNA and protein levels were significantly higher in tumor tissues from glioblastoma patients than in precancerous lesions. DGK $\zeta$ knockdown by lentivirus-delivered shRNA reduced glioblastoma cell proliferation and induced G0/G1 phase arrest. In addition, the rescue or overexpression of DGK $\zeta$ in glioblastoma cells further validated the function of DGK $\zeta$ as an oncogene. Moreover, knockdown of

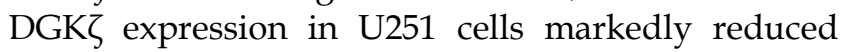
colony formation, endothelial cell tube formation in vitro and tumorigenic capability in vivo. At the molecular level, western blots showed that the

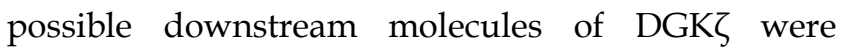
phosphorylated mTOR and AKT. In conclusion, these studies suggest that the suppression of DGK $\zeta$ could inhibit the tumor development of glioblastoma cells

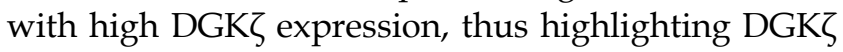
as a potential therapeutic target in malignant glioblastoma.

The underlying mechanism of DGK $\zeta$ inhibition of glioma cell growth was explored in this work. DAG and PA are important membrane components and biosynthetic precursors of phospholipids. DAG conversion into PA by DGK is the first step of replenishing PIP2 in the so-called "PIP2 cycle", a reaction that provides an important lipid substrate, not only for the subsequent hydrolysis by phospholipase C (PLC) but also for the production of PIP3 by the action of PI3 kinase (PI3K). This information indicated that DGK $\zeta$ might play important roles in the signaling pathways related to PIP2 or PI3K. Moreover, the PI3K/AKT/mTOR pathway has been regarded as an important signaling pathway that regulates many intracellular upstream signaling pathways that influence cell growth, metabolism, survival polarity, angiogenesis, and vesicle trafficking. In our study, AKT/mTOR was markedly inhibited by DKG $\zeta$ knockdown in U251 cells, indicating the interesting role of DKG $\zeta$ in glioma. Moreover, our results demonstrated that DGK $\zeta$ inhibition arrested the cell cycle at the G0/G1 phases. The AKT/GSK3 $\beta$ pathway induces cell cycle progression by enhancing cyclin D1 expression. The reason for this finding might be that these cells were screened during the process of tumor development, and some compensatory DGKろ-independent pathway was already activated in these screened cells to support cell survival and proliferation. However, the exact mechanisms require further investigation.

\section{Abbreviations}

DGKろ, diacylglycerol kinase zeta; shRNA, short hairpin RNA; DMEM, Dulbecco's modified Eagle's medium; FBS, fetal bovine serum; Scr-shRNA, scrambled shRNA; qRT-PCR, quantitative real-time PCR; CCK8, Cell Counting Kit 8; PI, propidium iodide; PIP2 cycle, phosphatidylinositol-4,5bisphosphatecycle; PI3K, phosphatidylinositol 3-kinase; AKT, V-akt murine thymoma viral oncogene homolog; mTOR, mammalian target of rapamycin.

\section{Acknowledgments}

This work was supported by the following funding: the Special Program for Collaborative Innovation, and Shanghai Municipal Education Commission (Class II Plateau Disciplinary Construction Program for Medical Technology of SUMHS, 2018-2020); the Seed fund of SUMHS; the Natural Science Foundation of Guangdong Province (2016A030313680).

The authors thank Vitaly Ryu for critical reading and editing of the manuscript.

\section{Competing Interests}

The authors have declared that no competing interest exists. 


\section{References}

1. Wen PY, Kesari S. Malignant gliomas in adults. N Engl J Med. 2008; 359: 492-507.

2. Koshy M, Villano JL, Dolecek TA, Howard A, Mahmood U, Chmura SJ, et al. Improved survival time trends for glioblastoma using the SEER 17 population-based registries. J Neurooncol. 2012; 107: 207-212.

3. Takahashi D, Suzuki K, Sakamoto T, Iwamoto T, Murata T, Sakane F. Crystal structure and calciuminduced conformational changes of diacylglycerol kinase a EF-hand domains. Protein Sci. 2019; 28: 694-706.

4. Baldanzi G. Inhibition of diacylglycerol kinases as a physiological way to promote diacylglycerol signaling. Adv Biol Regul. 2014; 55: 39-49.

5. Kanoh H, Yamada K, Sakane F. Diacylglycerol kinase: a key modulator of signal transduction? Trends Biochem Sci. 1990; 15: 47-50.

6. Han GS, O'Hara L, Siniossoglou S, Carman GM. Characterization of the yeast DGK1-encoded CTP-dependent diacylglycerol kinase. J Biol Chem. 2008; 283 : 20443-20453.

7. Topham MK, Bunting M, Zimmerman GA, McIntyre TM, Blackshear PJ, Prescott SM. Protein kinase $C$ regulates the nuclear localization of diacylglycerol kinase-zeta. Nature. 1998; 394: 697-700.

8. Brose N, Rosenmund C. Move over protein kinase C, you've got company: alternative cellular effectors of diacylglycerol and phorbol esters. J Cell Sci. 2002; 115: 4399-4411.

9. Kazanietz MG. Novel "nonkinase" phorbol ester receptors: the $\mathrm{C} 1$ domain connection. Mol Pharmacol. 2002; 61: 759-767.

10. English D. Phosphatidic acid: a lipid messenger involved in intracellular and extracellular signalling. Cell Signal. 1996; 8: 341-347.

11. Exton JH. Phosphatidylcholine breakdown and signal transduction. Biochim Biophys Acta. 1994; 1212: 26-42.

12. Hodgkin MN, Pettitt TR, Martin A, Michell RH, Pemberton AJ, Wakelam MJ. Diacylglycerols and phosphatidates: which molecular species are intracellular messengers? Trends Biochem Sci. 1998; 23: 200-204

13. Torres-Ayuso P, Tello-Lafoz M, Merida I, Avila-Flores A. Diacylglycerol kinase-zeta regulates mTORC1 and lipogenic metabolism in cancer cells through SREBP-1. Oncogenesis. 2015; 4: e164.

14. Fang Y, Vilella-Bach M, Bachmann R, Flanigan A, Chen J. Phosphatidic acid-mediated mitogenic activation of mTOR signaling. Science. 2001; 294: 1942-1945.

15. Brown JR, Auger KR. Phylogenomics of phosphoinositide lipid kinases: perspectives on the evolution of second messenger signaling and drug discovery. BMC Evol Biol. 2011; 11: 4.

16. Sakai H, Murakami C, Matsumoto KI, Urano T, Sakane F. Diacylglycerol kinase $\delta$ controls down-regulation of cyclin D1 for $\mathrm{C} 2 \mathrm{C} 12$ myogenic differentiation. Biochimie. 2018; 151: 45-53.

17. Torres-Ayuso P, Daza-Martin M, Martin-Perez J, Avila-Flores A, Merida I. Diacylglycerol kinase alpha promotes 3D cancer cell growth and limits drug sensitivity through functional interaction with Src. Oncotarget. 2014; 5: 9710-9726.

18. Takeishi K, Taketomi A, Shirabe K, Toshima T, Motomura T, Ikegami T, et al. Diacylglycerol kinase alpha enhances hepatocellular carcinoma progression by activation of Ras-Raf-MEK-ERK pathway. J Hepatol. 2012; 57: 77-83.

19. Dominguez CL, Floyd DH, Xiao A, Mullins GR, Kefas BA, Xin W, et al. Diacylglycerol kinase alpha is a critical signaling node and novel therapeutic target in glioblastoma and other cancers. Cancer Discov. 2013; 3: 782-797.

20. Nakano T, Iravani A, Kim M, Hozumi Y, Lohse M, Reichert E, et al. Diacylglycerol kinase eta modulates oncogenic properties of lung cancer cells. Clin Transl Oncol. 2014; 16: 29-35.

21. Guo Z, Jia J, Yao M, Kang J, Wang Y, Yan X, et al. Diacylglycerol kinase Y predicts prognosis and functions as a tumor suppressor by negatively regulating glucose transporter 1 in hepatocellular carcinoma. Exp Cell Res. 2018; 373: 211-220.

22. Kai M, Yamamoto E, Sato A, Yamano HO, Niinuma T, Kitajima H, et al. Epigenetic silencing of diacylglycerol kinase gamma in colorectal cancer. Mol Carcinog. 2017; 56: 1743-1752.

23. Mérida I, Torres-Ayuso P, Ávila-Flores A, Arranz-Nicolás J, Andrada E, et al. Diacylglycerol kinases in cancer. Adv Biol Regul. 2017; 63: 22-31.

24. Mérida I, Andrada E, Gharbi SI, Ávila-Flores A. Redundant and specialized roles for diacylglycerol kinases $a$ and $\zeta$ in the control of T cell functions. Sci Signal. 2015; 8: re6.

25. Cai K, Mulatz K, Ard R, Nguyen T, Gee SH. Increased diacylglycerol kinase zeta expression in human metastatic colon cancer cells augments Rho GTPase activity and contributes to enhanced invasion. BMC Cancer. 2014; 14: 208.

26. Tanaka T, Okada M, Hozumi Y, Tachibana K, Kitanaka C, Hamamoto Y, et al.

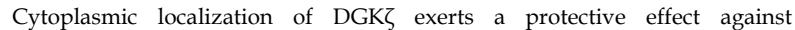
p53-mediated cytotoxicity. J Cell Sci. 2013; 126: 2785-2797.

27. Gu X, Wang C, Wang X, Ma G, Li Y, Cui L, et al. Efficient inhibition of human glioma development by RNA interference-mediated silencing of PAK5. Int J Biol Sci. 2015; 11: 230-237.

28. Sabates-Bellver J, Van der Flier LG, de Palo M, Cattaneo E, Maake C, Rehrauer H. Transcriptome profile of human colorectal adenomas. Mol Cancer Res. 2007; 5: 1263-1275.

29. Cerami E, Gao J, Dogrusoz U, Gross BE, Sumer SO, Aksoy BA et al. The cBio cancer genomics portal: an open platform for exploring multidimensional cancer genomics data. Cancer Discov. 2012; 2: 401-404.
30. Provenzani A, Fronza R, Loreni F, Pascale A, Amadio M, Quattrone A. Global alterations in mRNA polysomal recruitment in a cell model of colorectal cancer progression to metastasis. Carcinogenesis. 2006; 27: 1323-1333.

31. Ducker GS, Atreya CE, Simko JP, Hom YK, Matli MR, Benes CH. Incomplete inhibition of phosphorylation of $4 \mathrm{E}-\mathrm{BP} 1$ as a mechanism of primary resistance to ATP-competitive mTOR inhibitors. Oncogene. 2014; 33: 1590-1600.

32. Louis DN, Perry A, Reifenberger G, von Deimling A, Figarella-Branger D, Cavenee WK, et al. The 2016 world health organization classification of tumors of the central nervous system: a summary. Acta Neuropathol. 2016; 131: 803-820.

33. Pan Y, Yuan F, Li Y, Wang G, Lin Z, Chen L. Bromodomain PHD finger transcription factor promotes glioma progression and indicates poor prognosis. Oncol Rep. 2019; 41: 246-256.

34. [Internet] Roche. FDA grants Roche's Avastin full approval for most aggressive form of brain cancer. https://www.roche.com/investors/updates/inv-update-2017-12-06.htm

35. Karsy M, Guan J, Cohen AL, Jensen RL, Colman H. New Molecular Considerations for Glioma: IDH, ATRX, BRAF, TERT, H3 K27M. Curr Neurol Neurosci Rep. 2017; 17: 19.

36. Libermann TA, Nusbaum HR, Razon N, Kris R, Lax I, Soreq H, et al. Amplification, enhanced expression and possible rearrangement of EGF receptor gene in primary human brain tumours of glial origin. Nature. 1985; 313: 144-147.

37. Mohammad F, Weissmann S, Leblanc B, Pandey DP, Høifeldt JW, Comet I, et al. EZH2 is a potential therapeutic target for H3K27M-mutant pediatric gliomas. Nat Med. 2017; 23: 483-492.

38. Kwak Y, Kim SI, Park CK, Paek SH, Lee ST, Park SH. C-MET overexpression and amplification in gliomas. Int J Clin Exp Pathol. 2015; 8: 14932-14938.

39. Foltyn M, Luger AL, Lorenz NI, Sauer B, Mittelbronn M, Harter PN, et al. The physiological mTOR complex 1 inhibitor DDIT4 mediates therapy resistance in glioblastoma. Br J Cancer. 2019; 120: 481-487. 